\title{
Simultaneous Usage of NEMO and MANET for Vehicular Communication
}

\author{
Manabu Tsukada \\ INRIA Rocquencourt, IMARA \\ Team, Domaine de Voluceau, \\ BP 105, 78153 Le Chesnay \\ Cedex, France \\ manabu.tsukada@inria.fr
}

\author{
Olivier Mehani \\ INRIA Rocquencourt, IMARA \\ Team, Domaine de Voluceau, \\ BP 105, 78153 Le Chesnay \\ Cedex, France \\ olivier.mehani@inria.fr
}

\author{
Thierry Ernst \\ INRIA Rocquencourt, IMARA \\ Team, Domaine de Voluceau, \\ BP 105, 78153 Le Chesnay \\ Cedex, France \\ thierry.ernst@inria.fr
}

\begin{abstract}
Mobile Ad-hoc Network (MANET) routing protocols and NEMO Basic Support are considered key technologies for vehicle networks. Cooperation between MANET and NEMO (MANEMO) brings several benefits especially for route optimization and multihoming. We made a real field vehicle communication environment with NEMO and MANET. By switching from NEMO to MANET, routes between vehicles are optimized and network performances improved in terms of latency and bandwidth. Experiment results show that network performances are further improved with simultaneous usage of NEMO and MANET. Network performances were shown on a web site in real time and mapped at locations using GPS information.
\end{abstract}

\section{Keywords}

MANEMO, MANET, NEMO Basic Support, Multihoming, Vehicular network

\section{INTRODUCTION}

Road and vehicle circulation systems are one of the most important infrastructures and are supporting the humans' daily life. Intelligent Transportation Systems (ITS) aim to optimize the social costs of road systems and enhance their security as well as drivers' comfort by allowing such services as fleet management, navigation, billing, multimedia applications, etc. As communication technologies are considered the key point of ITS, mobility implemented in vehicles becomes crucial.

IPv6 is considered as able to fill ITS requirements thanks to its extended address space, embedded security, enhanced mobility support and ease of configuration. Thus future vehicles will embed a number of sensors and other IPv6enabled devices [1. When sensors deployed in vehicles are connected to the Internet and data from those is shared among vehicles, various applications can be considered: from speeds readings can be inferred valuable information on the

Permission to make digital or hard copies of all or part of this work for personal or classroom use is granted without fee provided that copies are not made or distributed for profit or commercial advantage and that copies bear this notice and the full citation on the first page. To copy otherwise, to republish, to post on servers or to redistribute to lists, requires prior specific permission and/or a fee.

WEEDEV 2008 Innsbruck, Austria

Copyright 200X ACM X-XXXXX-XX-X/XX/XX ...\$5.00. traffic conditions in real time; by reporting rapid brake activation of vehicles, dangerous segments can be advertised in advance; the road administrators can be informed about the dangerous parts of the road network before any accident happen; etc.

To support all of the ITS application requirements, onthe-move and uninterrupted Internet connectivity is necessary. Network Mobility (NEMO Basic Support, or NEMO for short [2]) has thus been specified by the IETF ${ }^{1}$ NEMO Working Group and recommended by the ISO TC204 WG16 draft standard (called $\mathrm{CALM}^{2}$ ) to achieve Internet mobility for vehicles.

Additionally, Mobile Ad-hoc Networks can be used for vehicular communication without depending on any thirdparty infrastructure. Several ad-hoc network routing protocols have been specified by the IETF Mobile Ad-hoc Networks (MANET) Working Group. These routing protocols are classified into the reactive or proactive groups. The $O p$ timized Link State Routing (OLSR) [3] protocol, used in the following, has been specified at IETF in the proactive group.

This paper is organized as follows. Network technologies related to vehicle communication are summarized in section 22, then section 3 addresses scenario and objectives of this study. Our implementation on Linux is mentioned in section 4, and the demonstration of the system in the IPv6 mobility testbed of the ANEMONE [4] project is described in section 5 . Network performances have been evaluated in both an indoor and real field testbeds, which results are presented in section 6. Section 7. concludes this papers summarizing the results, and stating future works to be undergone.

\section{TECHNOLOGIES FOR VEHICLE COM- MUNICATION}

In this section, works related to vehicle communication using NEMO and MANET, such as Multihoming, Route Optimization and MANEMO, are summarized.

\subsection{NEMO}

The NEMO Basic Support functionalities involve a router on the Internet to allow mobile computers to communicate with other nodes, be they mobile or static. In light of the ITS field of application, the basic scheme is as follows. A Mobile Router (MR) located in the vehicle acts as a gateway for the Mobile Network of the vehicle, and manages mobility

${ }^{1}$ IETF: Internet Engineering Task Force

${ }^{2}$ CALM: Air interface, Long and Medium range 
on behalf of its Mobile Network Nodes (MNN). The MR and a fixed router in the Internet called the Home Agent (HA) establish a bi-directional tunnel to each other which is used to transmit the packets between the MNN and their Correspondent Nodes (CN).

\subsection{Multihoming}

Mobile Routers can be shipped with multiple network interfaces such as IEEE802.11a/b/g, WiMAX, GPRS/UMTS, etc. When an MR maintains these interfaces simultaneously up and has multiple paths to the Internet, it is said to be multihomed. In mobile environments, MRs often suffer from scarce bandwidth, frequent link failures and limited coverage. Multihoming brings the benefits of alleviating these issues [5]. The possible configurations offered by NEMO are classified in 6], according to three parameters: (x) the number of MRs in the mobile network, (y) the number of HAs serving the mobile network, and ( $\mathrm{z}$ ) the number of MNPs (Mobile Network Prefixes) advertised in the mobile network. In this paper, we focus on the "single MR, single HA and single MNP" configuration, referred to as $(x, y, z)=(1,1,1)$.

In this configuration, a tunnel is established between the HA address and a Care-of Address (CoA) of the MR in NEMO Basic Support, even if the MR is equipped with several interfaces. Multiple Care-of Addresses Registration (MCoA) 7 is thus proposed as an extension of both Mobile IPv6 and NEMO Basic Support to establish multiple tunnels between MR and HA. Each tunnel is distinguished by its Binding Identification number (BID). In other words, NEMO Basic Support only realizes interface switching while MCoA supports simultaneous use of multiple interfaces.

\subsection{Route Optimization}

NEMO is one of key technologies of vehicle communication, however, issues related to Route Optimization still remain in NEMO Basic Support, while they already have been solved in Mobile IPv6 8]. In NEMO, all the packets to and from MNNs must be encapsulated with IPs in the tunnel between the MR and the HA. Thus all these packets between MNNs and CNs must go through the HA. This causes various problems and performance degradation. These suboptimal effects are described as follows.

Suboptimal routes are caused by the packets being forced to pass by the HA. This leads to increased delay that is undesirable for applications such as real-time multimedia streaming. Packet Encapsulation of additional 40 bytes header increases packets overhead and risks of packet fragmentation. This results in an increased processing delay for every packets being encapsulated and decapsulated in both the MR and the HA. Bottlenecks in the HA are a severe issue because significant traffic to and from MNNs is aggregated in the HA when it supports several MRs acting as gateways for several MNNs. This may cause congestion at the HA that would lead to additional packet delays, or even packet losses. Nested Mobile Networks is an issue that NEMO Basic Support raises by having arbitrary levels of nesting of mobile networks. This permits an MR to host other MRs in its mobile network. With nested mobile networks, the use of NEMO further amplifies the sub-optimality listed above.

In IETF, the issues of Route Optimization of NEMO are addressed in 9 and the solution space is analyzed in [10. Requirements of Route Optimization in various scenarios are described for networks for vehicles 11 and aeronautic environments [12.

\subsection{MANEMO}

Both MANET and NEMO are designed independently as Layer 3 technologies. NEMO is designed to provide global connectivity and MANET to provide direct route in local area network. MANEMO that is the concept of using both MANET and NEMO together could bring benefits of route optimization.

Since direct routes are available in MANET local networks, MANET can provide direct paths between vehicles. These paths are optimized and free from the NEMO tunnel overhead 13, 14. The possible topology configuration with MANEMO is described in 15] and issues and requirements are summarized in [16]. In addition, MANEMO are used for vehicle communication, for example, VARON 17. focuses on NEMO route optimization using MANET. It also provides the same level of security as the current Internet even if the communication is done via the MANET route.

\section{SCENARIO AND OBJECTIVES}

In this paper, we focus on the scenario of inter-vehicle communication shown in Figure 1. Sensors fixed in the vehicle are connected to the Internet to share the vehicle's information, and fixed PCs or mobile terminals brought by the passengers are connected to the mobile network in the vehicle. Vehicles are connected to the Internet everywhere and anytime with multiple interfaces by using NEMO. The MR acting as a gateway of the mobile network has both NEMO and MANET connectivity.

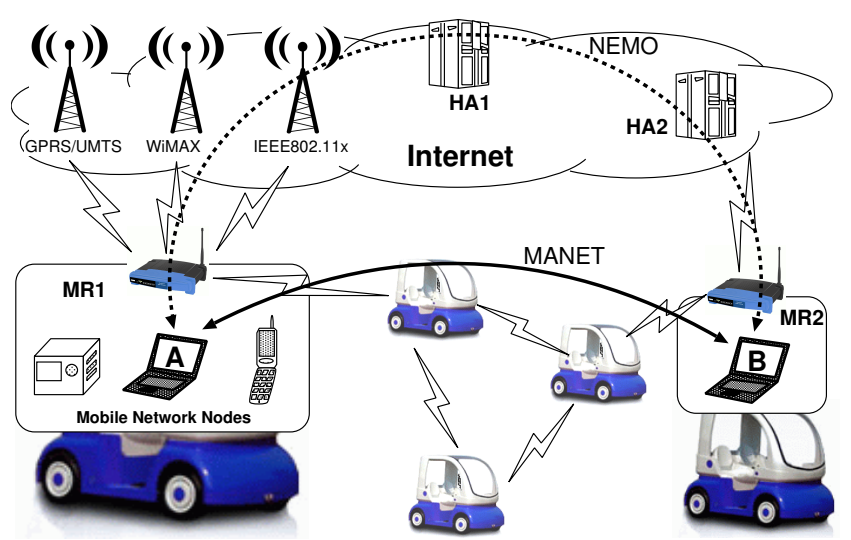

Figure 1: Experimentation Scenario.

In preceding works, switching from a NEMO route to a MANET route brought the benefits of route optimization in terms of bandwidth and delay. In this paper, we propose simultaneous utilization of the NEMO and MANET routes. Traffic distribution into multiple paths can contribute to further improvement of communication performances.

The objectives are to investigate MANEMO. We set a testbed up in a real field environment using four-wheeled electric vehicles (CyCabs [18) to discover issues and requirements in real environments. Real field testbed helped us to prepare a feasible study considering wireless links characteristic, link change, vehicle movement, etc. 
The testbed has three aims. First, MANEMO has a possibility to improve network performances by allowing Route Optimization via simultaneous usage of NEMO and MANET. Network performances can be evaluated in this testbed. Second, we have plans to develop vehicle communication applications. Applications can be developed and validated within the testbed environment. Third, demonstrations can be performed to show all of the works (all layers) together. We demonstrated MANEMO technologies at the EuropeanCommission-funded R\&D project called ANEMONE, which aims at realizing a large-scale testbed for mobility technologies, in December 2007. Our previous works on MANEMO are described in 19 .

\section{DESIGN AND IMPLEMENTATION}

In this section, design and implementation are mentioned.

\subsection{Policy routing}

The system is implemented on GNU/Linux (kernel 2.6.21.3). To distribute packets to multiple paths simultaneously in the MR, policy routing is used. Classic routing mechanisms are not suitable, because of the "longest match" principle. As shown in Fig. 2 the packets arriving to the MR are matched with the routing table entries. In the MANEMO case, MANET routes typically have longer prefix lengths than NEMO ones. The former can thus be used in priority when present in the routing table. The NEMO routes may have the least preference as default routes. A single routing table can be used for switching between routes, but not for simultaneous usage of NEMO and MANET.

We thus propose to introduce multiple routing tables using Route Policy Database (RPDB) to the system as shown in Fig. 3 To achieve this goal, the network filtering framework called Netfilter ${ }^{[3}$ is used. The RPDB allows to maintain several independent routing tables in the kernel. Each packet can then be routed according to one of these tables. The determination of which routing tables should be used in a particular case is up to the implementer. It is usual to route depending on the type of flow that is being routed. This mechanism allows distributing packets to multiple routes at the same instant.

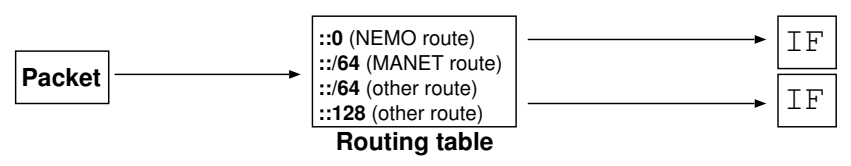

Figure 2: Classic Routing.

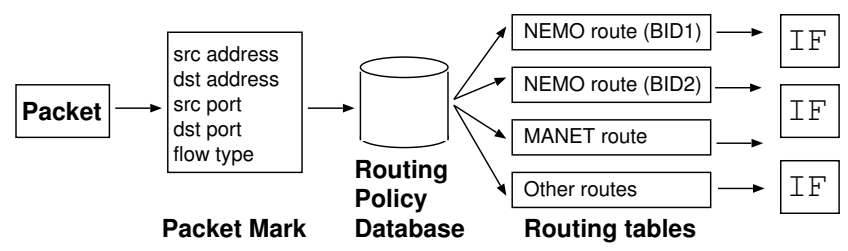

Figure 3: Multiple Routing Tables.

\footnotetext{
$\sqrt[3]{\text { http://www.netfilter.org }}$ standard packet manipulation stack as of the 2.6 series.
}

\subsection{Implementation Details}

NEPL (NEMO Platform on Linux, version 20070716) ${ }^{4}$ has been installed on the MR as well as an OLSR daemon from http://www.olsr.org (version 0.5.3). NEPL is developed and distributed freely by the Nautilus6 project ${ }^{5}$ within the WIDE project 6 . NEPL is based on MIPL (Mobile IPv6 for Linux) ${ }^{7}$ developed in the Go-Core (Helsinki University of Technology) and Nautilus6 projects.

The OLSR daemon has been adapted to the routing scheme proposed in section 4 . The modifications were so that the OLSR routing entries are maintained in one of the multiple routing tables of the kernel. The NEMO daemon already handles policy routing when patched for MCoA support 8 .

On the MR, the NEMO and OLSR daemons are working independently. The NEMO daemon maintains its binding update list and NEMO routes while the OLSR daemon takes care of MANET routes. As shown in Fig. 4 both NEMO and MANET routing entries are kept up-to-date in separate tables.

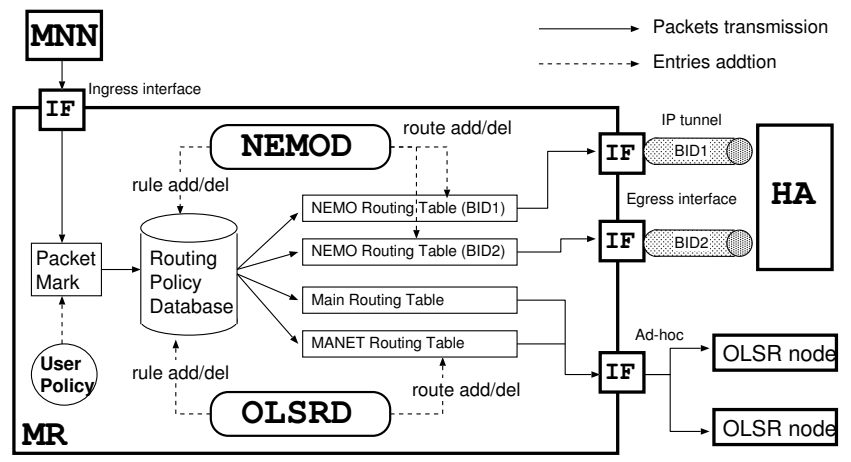

Figure 4: Overview of the System.

When started, both daemons add the rule entries that tell which packets should be routed according to which routing table (they are removed at the daemons' termination). The MR has multiple routing tables that are allocated to the NEMO, MANET and MAIN routes. There is the same numbers of NEMO routing tables as of egress interfaces on the MR. Each routing table is assigned a specific BID. The MANET routing table is used for traffic that should be routed directly to other vehicles, and the MAIN table is mostly used to route OLSR signaling. The route entries of the NEMO routing tables are added and removed by the NEMO daemon whereas those of the MANET routing table are added and removed by the OLSR daemon.

The packets from the MNNs arrive at the MR with source and destination addresses and ports as well as flow type information. The packets are distributed, according to the latter mark, using either of the NEMO or MANET routing tables. The packets destined to the NEMO routing table are transmitted to the tunnel bound to the HA. The packets coming in for MANET routing are transferred to other OLSR nodes directly.

${ }^{4}$ http://software.nautilus6.org/NEPL-UMIP/

${ }^{5}$ http://www.nautilus6.org

${ }^{6}$ http://www.wide.ad.jp

7 http://www.mobile-ipv6.org

'http://software.nautilus6.org/MCoA/ 


\section{DEMONSTRATION SYSTEM}

The system has been demonstrated in real field during the Promotion Day for the ANEMONE project 12th-14th December 2007 as an example third party experiments using the mobility testbed.

\subsection{Network Overview}

Fig. 5 shows an overview of the network configuration of the testbed. Each MR is installed in a vehicle. MR1 has four interfaces: Ethernet, IEEE802.11b ad-hoc mode, IEEE802.11b managed mode, and 3G (6in4 OpenVPN tunnel over a PPP link provided by SFR), MR2 has the same interfaces except for 3G. MR1 is supported by HA1 in INRIA Rocquencourt network. MR2 is supported by HA2 in IRISA's network. Both networks are located in France and interconnected via RENATER (French backbone for education and research) using a direct 6 in 4 tunnel to work around some IPv6 routing problems (the testbed sites are $12 \mathrm{IPv} 4$ hops apart). MNN1 is a MAC OSX $10.4 \mathrm{PC}$ and MNN2 is a Windows XP tablet PC, the other nodes appearing in Fig. 5 run Linux.

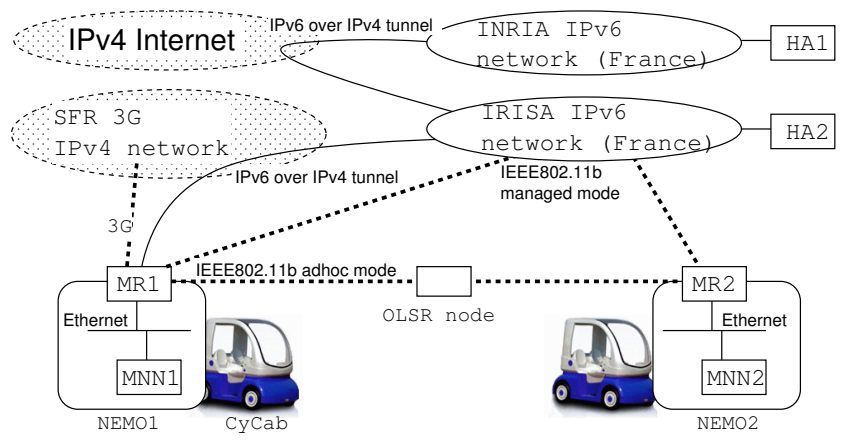

Figure 5: Network Topology of System.

The parameters of the OLSR algorithm had to be tuned down (Table 1) to accommodate to the high and fast mobility of a vehicular network. These modifications allow the MRs to discover others' becoming (un)available more quickly.

Table 1: OLSR Parameters in Use.
\begin{tabular}{|c|cc|}
\hline Parameter & Value & (default) \\
\hline \hline HELLO interval & $0.5 \mathrm{sec}$ & $(2.0 \mathrm{sec})$ \\
\hline HELLO validity & $1.5 \mathrm{sec}$ & $(6.0 \mathrm{sec})$ \\
\hline HNA interval & $1.0 \mathrm{sec}$ & $(5.0 \mathrm{sec})$ \\
\hline HNA validity & $3.0 \mathrm{sec}$ & $(15.0 \mathrm{sec})$ \\
\hline
\end{tabular}

Radio interferences between IEEE $802.11 \mathrm{~b}$ managed and ad-hoc networks were experienced even if channels frequencies were well apart from one another. To overcome the problem, the bandwidths of the Wi-Fi interfaces on MR1 were limited to 2 Mbits/sec using Linux' QoS system based on tc (Traffic Control) 9 .

Network performances between MNNs are summarized in Table 2

${ }^{9}$ http://www.linux-foundation.org/en/Net:Iputils
Table 2: Network Performances Between MRs.

\begin{tabular}{|l|r|r|}
\hline Interface & RTT & Bandwidth \\
\hline \hline NEMO on 3G & $279.43 \mathrm{~ms}$ & $416 \mathrm{Kbits} / \mathrm{sec}$ \\
\hline NEMO on $802.11 \mathrm{~b}$ managed & $32.74 \mathrm{~ms}$ & $1977 \mathrm{Kbits} / \mathrm{sec}$ \\
\hline OLSR on $802.11 \mathrm{~b}$ Ad-hoc & $8.58 \mathrm{~ms}$ & $1987 \mathrm{Kbits} / \mathrm{sec}$ \\
\hline
\end{tabular}

\subsection{Demonstration Overview}

As a demonstration, network performances between two vehicles are shown on a website, mapped to their geographical positions. Measurements were made with a GPS-enabled Iper 10 in a topology as shown in Fig. 6. MNN1 works as an Iperf server and MNN2 is the client. Iperf reports the size of transferred data and used bandwidth. Additionally, the GPS patch appends location information of the MNNs (latitude and longitude), and the offset and distance from the starting point. Only a regular GPS (serial, USB, Bluetooth, etc.) is needed.

The demonstration can be performed in either real time mode or log mode. The former shows network performances mapped with position in the real time on the web site while the latter records them on the MMN's local disk to be displayed on the web site afterwards, with the measurements. The website could initially be seen from the IPv4 Internet only, because of Google Maps' currently only being available in IPv4. Using an IPv6/IPv4 web proxy, the site can be seen from the entire IPv6 network, including from the vehicles.

In real time mode, XML files are generated from measured performance and position every 2 seconds in MNN1. An example of XML output is shown in Fig. 7 The remote web server gets the XML file from MNN1 every 2 seconds using wget. Real time mode has the advantage that everyone can see network performances live, however the measurement can be slightly affected by the XML file transfers, as it goes through NEMO route. In contrast, log mode does not affect the measures. The results shown in this paper were taken in $\log$ mode. The network performance between the vehicles is available on the web site 11 . In addition, a screenshot of the website appears in section 6.4

\section{EVALUATION}

Basic measurements of latency and bandwidth were taken both in an indoor and a real field testbed. The results were mapped with position information on the website to be analyzed.

\subsection{Test Scenario}

Network performances were evaluated in the indoor testbed to avoid interferences due to unexpected radio perturbations and difficulties to trace the movements of the MRs. The following experiments were performed outside of any vehicles. Both the MRs and MNNs did not actually move during the 300 seconds experiments.

From $t=0$ to $t=60$, both Wi-Fi managed and ad-hoc interfaces equipped on MR 1 are down. At $t=60$, the managed interface comes up. At $t=120$, the ad-hoc one becomes

\footnotetext{
10 http://gforge.inria.fr/frs/?group_id=620\&release_ id=915

${ }^{11}$ http://fylvestre.inria.fr/ ${ }^{\sim}$ tsukada/experiments/
} 


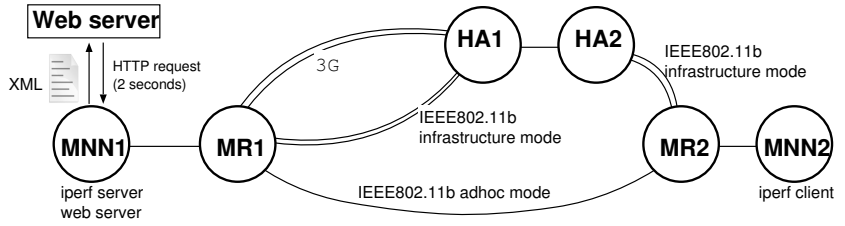

Figure 6: Network Topology of the Iperf Test System.

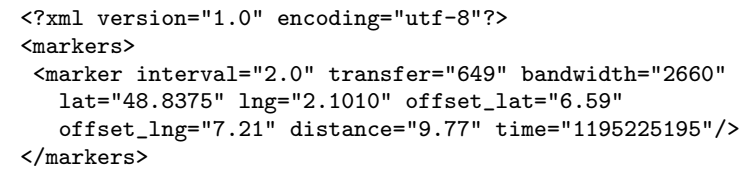

Figure 7: XML Data Generated from Iperf Results.

available. From $t=120$ to $t=180$, all the interfaces are up and running. At $t=180$, the ad-hoc link is taken down. At $t=240$, the managed one is also shut down. The $3 \mathrm{G}$ interface is always available in the test. These operations are performed using a shell script and the usual system tools.

MNN1 has three addresses $(A, B$ and $C)$ in the MNP and MR1 distributes traffic from the mobile network via multiple paths depending on the source address. Flow distribution policies are pre-configured on MR1 as listed in Table 3 The numbers in the table are priorities when multiple paths are available on MR1. The smallest value has the highest priority.

MR1 always forwards the packets from source address $A$ and to port number 5102 via the $3 \mathrm{G}$ interface. The packets from source address $B$ to destination port 5101 are routed via the Wi-Fi managed mode interface, when available. Otherwise, the packets are forwarded via the $3 \mathrm{G}$ interface. The packets from source address $C$ or to destination port number 5009 are transmitted via whatever interface is available with a priority order as listed in Table 3 HA1 distributes flows into the managed mode interface and $3 \mathrm{G}$ as per the second policy of MR1. MR2 distributes flows into managed and ad-hoc interfaces as of the third policy of MR1 except for the $3 \mathrm{G}$ interface.

Table 3: Flow Distribution Policy for the First Mobile Router.

\begin{tabular}{|c|c|c|c|c|}
\hline Policy & Targets & 3G & managed & Ad-hoc \\
\hline \hline Always 3G & Src $A$ or Dst port 5102 & 1 & $\times$ & $\times$ \\
\hline 3G + managed & Src $B$ or Dst port 5101 & 2 & 1 & $\times$ \\
\hline All interfaces & Src $C$ or Dst port 5009 & 3 & 2 & 1 \\
\hline
\end{tabular}

\subsection{Latency Measurements}

To measure the Round Trip Time (RTT) between MNNs, MNN1 sent 56 Bytes ICMPv6 Echo Request packets from the three addresses $(A, B$ and $C$ ) to MNN2 once in 0.5 sec. There was no traffic other than ping6. These packets were distributed according to the policies described in section 6.1. Fig. 8 shows the result. The average RTT on the NEMO route over $3 \mathrm{G}$ was $261.9 \mathrm{~ms}$. Changing paths to the
NEMO route over the managed Wi-Fi interface, the RTT reduced to $34.72 \mathrm{~ms}$ on average, which a represents an $87 \%$ improvement. The RTT on the OLSR route (ad-hoc link) was $7.93 \mathrm{~ms}$. The route optimization by MANEMO further reduced the latency by $26.79 \mathrm{~ms}$ which a represents a $77 \%$ improvement.

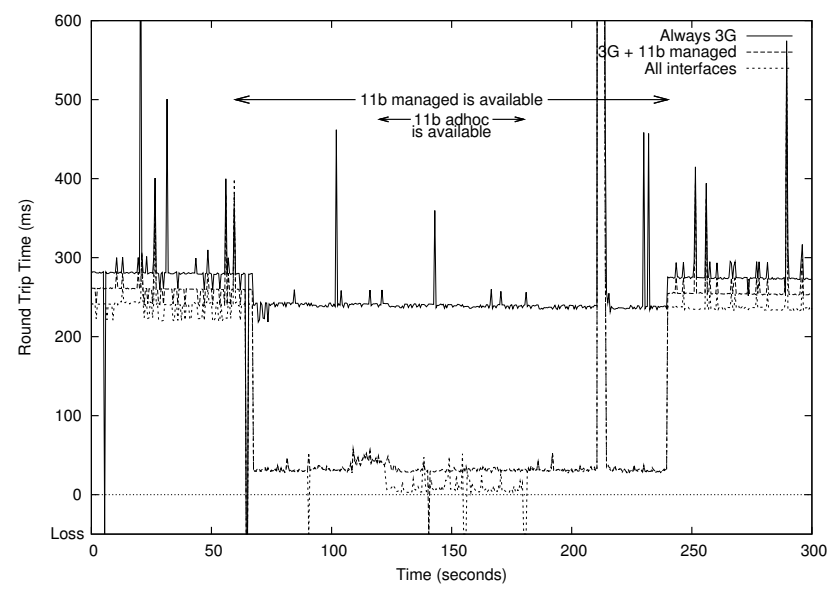

Figure 8: Round Trip Time Without any Other Traffic.

Fig. 9 gives a closer look at the route change periods, which is when the ad-hoc interface comes up and goes down. At $t=120$, the ad-hoc interface comes up, and then direct route information of both MNPs are exchanged. At $t=$ 122.5 , the RTT of the packet marked $21.27 \mathrm{~ms}$ which is an average RTT over the NEMO and OLSR routes. This is because either the out- or inbound route still uses NEMO while the other is already the MANET one. It takes $2.5 \mathrm{sec}$ for OLSR routing entries to be added to MR1's routing table after the ad-hoc link has been connected. In contrast, the route was changed back from OLSR to NEMO $1.5 \mathrm{sec}$ after the ad-hoc mode link was disconnected. While switching routes, 3 packets are lost (From $t=180$ to $t=181.5$ ).
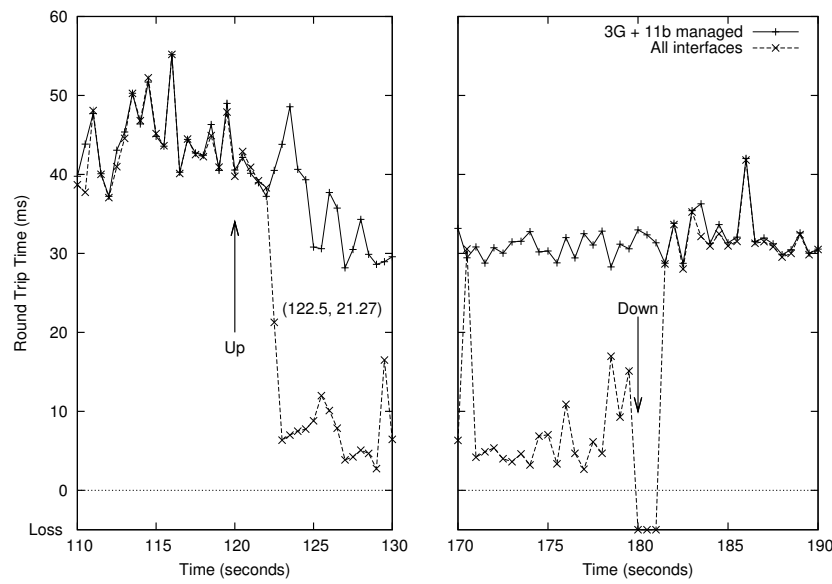

Figure 9: Closer Look of the Round Trip Time.

\subsection{Throughput Measurements}


To measure the throughput between MNNs, MNN1 sends 3 TCP streams to MNN2 with port numbers 5102, 5101 and 5009 using Iperf in the routing scenario described in section 6.1. At the same time, MNN1 also sends 56 Bytes ICMPv6 Echo Request packets as in section 6.2. Iperf gives a report once every $2 \mathrm{sec}$ and ping 6 gives its once in $0.5 \mathrm{sec}$. Fig. 10 shows the result of the throughput and Fig. 11] shows the RTT while the TCP traffic transmitted.

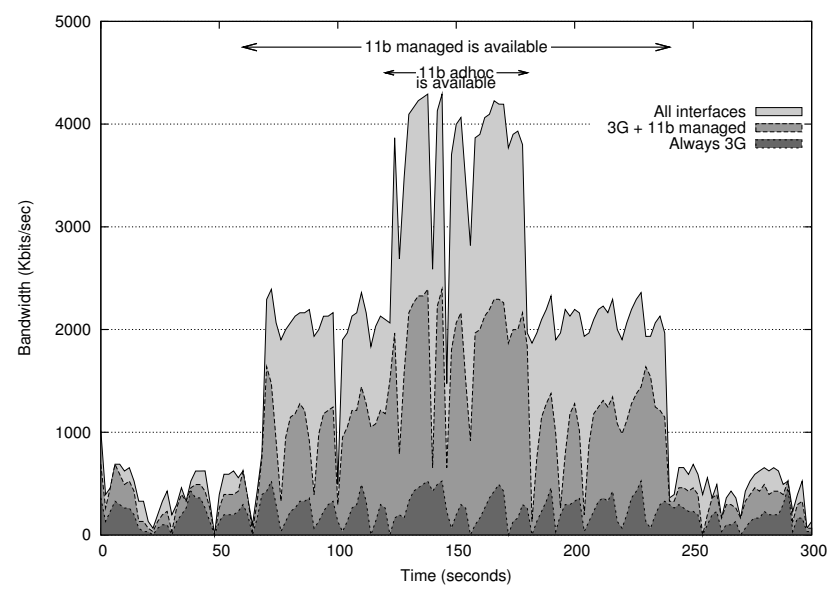

Figure 10: Throughput Between MNNs with 3 TCP Streams.

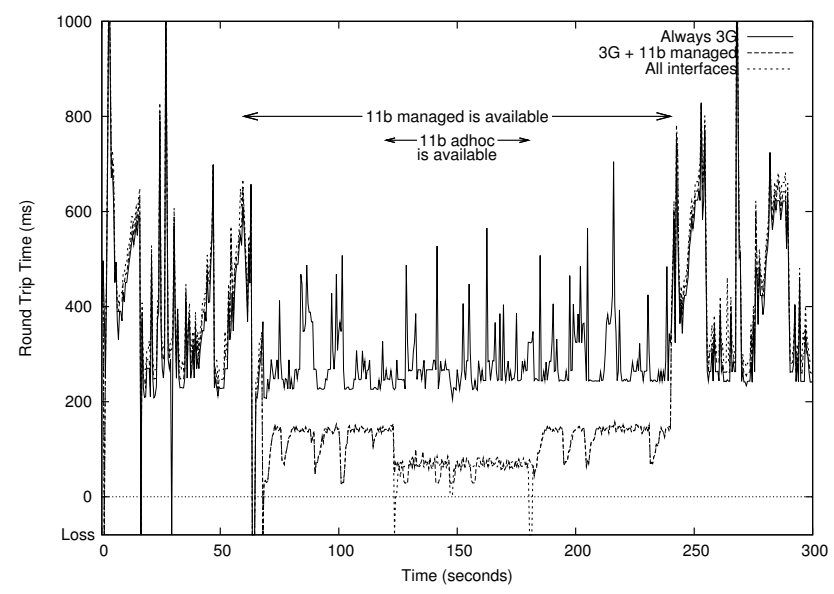

Figure 11: Round Trip Time Between MNNs During the Transmission of 3 TCP Streams.

The total throughput is listed in Table 4. The average throughput on the NEMO route over $3 \mathrm{G}$ is $455 \mathrm{Kbits} / \mathrm{sec}$ in total for 3 TCP streams from $t=0$ to $t=60$. Since an IEEE $802.11 \mathrm{~b}$ managed network is available and the flows are distributed to two paths from $t=60$ to $t=120$, the average throughput increases to a total of $1913 \mathrm{Kbits} / \mathrm{sec}$, which a represents a $76 \%$ (1458 Kbits/sec) improvement. From $t=120$ to $t=180$, in addition, ad-hoc connectivity is also available. Three TCP flows being distributed to 3 paths, the total average throughput increases to $3752 \mathrm{Kbits} / \mathrm{sec}$, which represents a $49 \%$ (1837 Kbits/sec) improvement.

The average RTT between MNNs is also listed in Table 4 . The RTT on the NEMO route with $3 \mathrm{G}$ is about $400 \mathrm{~ms}$ when all of the 3 TCP streams are transmitted using the $3 \mathrm{G}$ link.
Table 4: Throughput and RTT Between MNNs.

\begin{tabular}{|c|r|r|r|}
\hline \multirow{2}{*}{ Policy } & \multicolumn{3}{|c|}{ Available Interfaces } \\
\cline { 2 - 4 } & Only 3G & $3 \mathrm{G}+$ managed & All interfaces \\
\hline \hline \multicolumn{4}{|c|}{ Throughput } \\
\hline \hline Always 3G & $156 \mathrm{Kbps}$ & $262 \mathrm{Kbps}$ & $276 \mathrm{Kbps}$ \\
3G + managed & $184 \mathrm{Kbps}$ & $733 \mathrm{Kbps}$ & $1612 \mathrm{Kbps}$ \\
All interfaces & $114 \mathrm{Kbps}$ & $918 \mathrm{Kbps}$ & $1863 \mathrm{Kbps}$ \\
\hline Total & $455 \mathrm{Kbps}$ & $1913 \mathrm{Kbps}$ & $3752 \mathrm{Kbps}$ \\
\hline \hline \multicolumn{4}{|c|}{ Round Trip time } \\
\hline \hline Always 3G & $389 \mathrm{~ms}$ & $277 \mathrm{~ms}$ & $275 \mathrm{~ms}$ \\
3G + managed & $411 \mathrm{~ms}$ & $127 \mathrm{~ms}$ & $64 \mathrm{~ms}$ \\
All interfaces & $432 \mathrm{~ms}$ & $130 \mathrm{~ms}$ & $64 \mathrm{~ms}$ \\
\hline
\end{tabular}

When 2 TCP streams are diverted to the IEEE802.11b managed mode interface from $t=60$ to $t=120$, the RTT on $3 \mathrm{G}$ decreases by about $280 \mathrm{~ms}$, which represents a $30 \%$ improvement. The RTT also decreases from about $400 \mathrm{~ms}$ to $130 \mathrm{~ms}$ with policy " $3 \mathrm{G}+$ managed" and "All interfaces" which is a $68 \%$ improvement during the period when a managed network is available. In addition, a further $50 \%$ (approx.) improvement is recorded with policy " $3 \mathrm{G}+$ managed" and "All interfaces", when all the interface on MR1 are available.

\subsection{Real field Evaluation}

The system has been evaluated in a real field testbed on ENST-Bretagne/INRIA Rennes' campus. 40 access points were available in the area. The vehicle with MR2 parked beside a building which has 2 access points and the vehicle with MR1 moved around the building at the speed of about $10 \mathrm{~km} / \mathrm{h}(2.8 \mathrm{~m} / \mathrm{sec})$. The MRs were mounted inside of the vehicle. Three TCP streams were transmitted from MNN1 to MNN2 as in section 6.3 The flow distribution policies of MR1, MR2, HA1 and HA2 were also identical to those of the indoor testbed.

On Fig. 12, the switch between access medium and/or networks has a clear impact on the available bandwidth. From $t=0$ to $t=60$, the path between MNNs was only via NEMO route over $3 \mathrm{G}$. The average throughput of $3 \mathrm{TCP}$ was $344 \mathrm{Kbits} / \mathrm{sec}$ during this period. The throughput on real field was $111 \mathrm{Kbits} / \mathrm{sec}$ less than that of the indoor testbed due to obstacles and movements of the vehicle. From $t=62$ to $t=86$ and from $t=106$ to $t=116$, the NEMO route on the managed mode interface was available. The average throughputs of $3 \mathrm{TCP}$ streams are $1430.83 \mathrm{Kbits} / \mathrm{sec}$ and $957.34 \mathrm{Kbits} / \mathrm{sec}$, respectively. From $t=124$ to $t=130$, the OLSR route on via the MANET was available. The average throughput was $2408.4 \mathrm{Kbits} / \mathrm{sec}$.

In the evaluation, the NEMO route on the IEEE802.11b managed interface could be used during 24 seconds, then an additional 10 seconds. As the speed of the vehicle was 2.8 $\mathrm{m} / \mathrm{sec}$, the coverage of the access point can be estimated to a 30 to 65 meters area. The ad-hoc mode interface was available during 6 seconds, the available area can be estimated to 17 meters. The antennae of both MRs were located inside of the vehicle. The areas could be extended by using more powerful antennae and mounting them outside of the vehicle.

\subsection{Position-Mapped Network Performances}




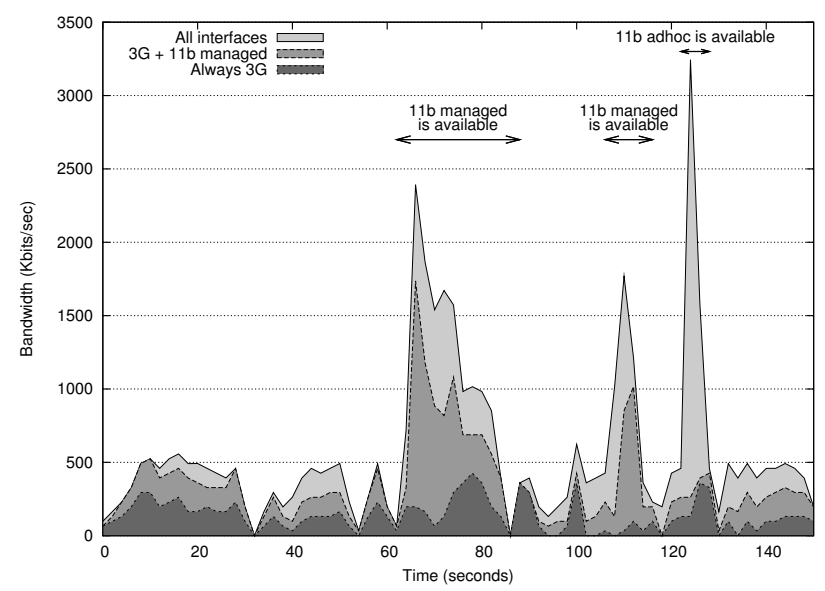

Figure 12: Throughput Variation Depending on the Available Connectivity.

Measured throughputs using a GPS-enabled Iperf between MNNs are rendered on a website ${ }^{[12}$ using the Google Maps API. The throughputs were measured at INRIA Rocquencourt in France. MR1 and MNN1 moved around, while MR2 and MNN2 stood still on the position indicated on the screenshot. The evaluation was actually performed without vehicles. A human with MR1 moved around in the area 65 meters from the position of MR2. It was carried by a human to obtain high density of throughput data around MR2, as the average speed of movement about $4 \mathrm{~km} / \mathrm{h}$. He started from the position of MR2 and came back to the same position in about 250 seconds.

Both MRs have IEEE802.11b managed and ad-hoc mode interfaces. No MR went out of the access points' coverage. As the wireless access points were quite close to the test site, the managed interface has been forcibly limited to $1 \mathrm{Mbits} / \mathrm{sec}$ to account for more distant APs and better highlight which network path MR1 used. In contrast, the ad-hoc interface was not limited and the average throughput between MRs using this interface was $2685 \mathrm{Kbits} / \mathrm{sec}$. The experiments were run 8 times and all the results are available on the aforementioned website.

The website (Fig. 13 is a screenshot where an OLSR node can also be seen; it was a static network node in the MANET) shows the throughput between MNNs varying the size of the blue circles at each measure point. All of the 8 evaluations can be seen by selecting the Log option. Clicking on one of the circles reveals information including the time from start, location and offset from the starting point, transfer data size and bandwidth in the last 2 seconds. All the data can be shown at once with the "Show Log" button. Users can also analyze the data by changing data density and see the trajectory of the MR1 and MNN1.

Fig. 14 shows the throughput statistics depending on the distance between MNNs. The points show the actual throughput during the experiments while the bar graph represents an average over 5 meters. Values over $1 \mathrm{Mbits} / \mathrm{sec}$ (the arbitrary limitation on the managed Wi-Fi link) had to be recorded because those were when the network path in use was the MANET. One can see that the OLSR route is avail-

\footnotetext{
${ }^{12}$ http://fylvestre.inria.fr/ ${ }^{\sim}$ tsukada/experiments/
}

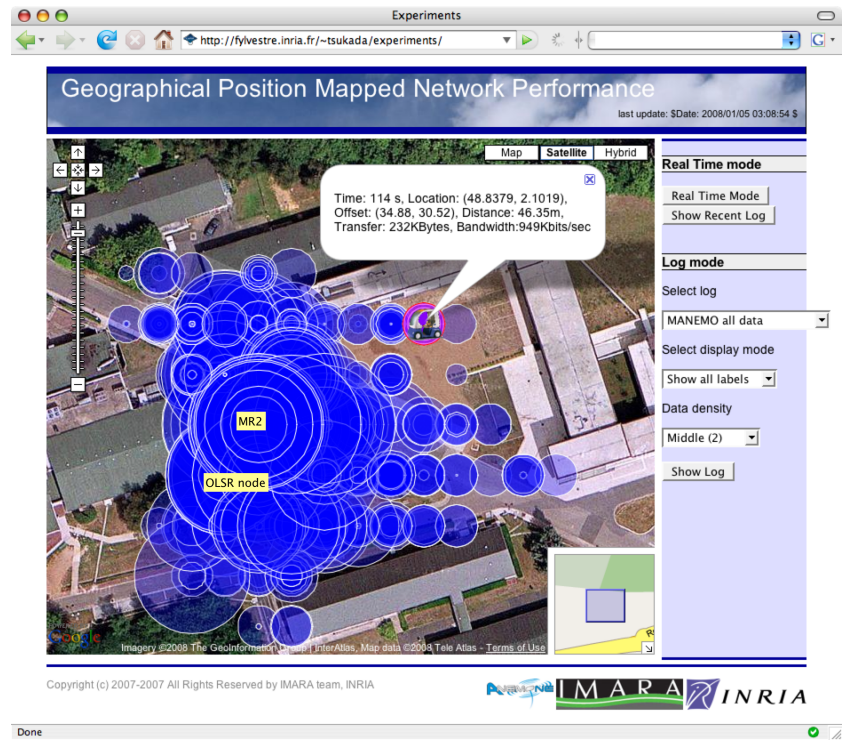

Figure 13: Screenshot of the Report Website.

able up to 40 meters. Between (approx.) 20 and 40 meters, the throughputs spread over a wide range from $100 \mathrm{Kbits} / \mathrm{sec}$ to $2700 \mathrm{Kbits} / \mathrm{sec}$, because media handovers were performed between the managed and ad-hoc interfaces in these zones.

An asymmetrical tendency of the ad-hoc link range has been observed. From the gathered results, it turns out that the OLSR route is available over a longer distance when two vehicles are getting farther from one another than when they are getting closer. This hysteresis behavior is due to OLSR's initial delay caused by the sending period for HELLO packets.

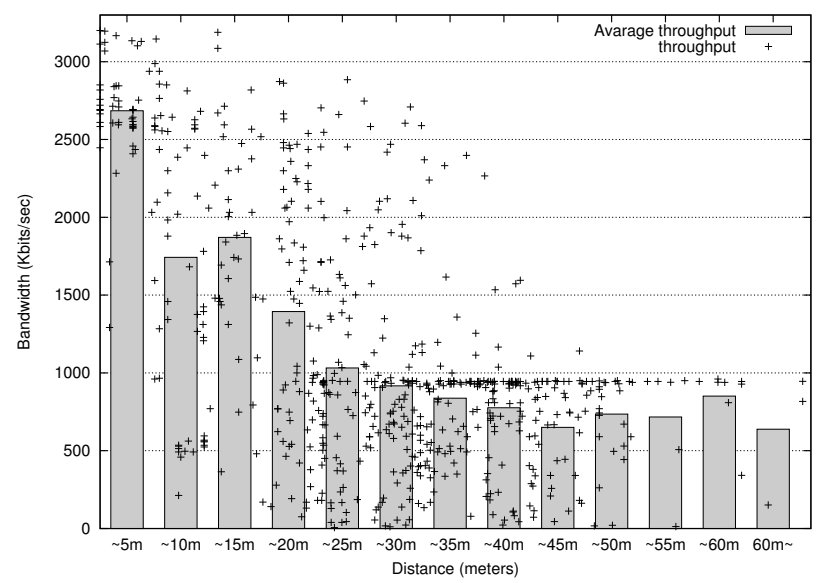

Figure 14: Decrease in Bandwidth with the Increase of Distance.

\section{CONCLUSION AND FUTURE WORKS}

We have set up an experimental environment to investigate MANEMO using CyCabs in a real field. In our system, the MRs utilize multiple egress interfaces simultaneously 
with NEMO and/or OLSR. OLSR can mitigate the suboptimality caused by NEMO. Previous experiments results showed that MANEMO with route switching from NEMO to MANET can improve network performance in terms of network latency and bandwidth. It can now be stated that MANEMO, with simultaneous usage of NEMO and MANET, can achieve further improvements of the network performances. The experimental results show that throughputs and latencies improved when all interfaces $-3 \mathrm{G}$ and both IEEE 802.11b managed and ad-hoc - were available.

We furthermore plan to develop a MANEMO real field environment on the CyCabs as follows. First, the evaluation results show that network performances such as latency and bandwidth dynamically change according to the available interfaces, movement or obstacles. Thus adaptive applications are desired in these environments. Second, the flows have to be allocated to appropriate paths concerning about application demand and network performance. Since realtime applications are sensitive for handover, intelligent path allocation is required. Third, the traffic between MNNs were distributed according to the policies specified by the administrator. As an MR can determine only outbound traffic, policy changes on an MR may create asymmetric routes. By introducing common filter rules and exchanging them among MRs and HAs 20, 21, the policy on each entity could be kept in sync. Fourth, the position-mapped network performances report currently shows only bandwidth. Other network performances such as latency, packet loss rate or L2 information would be useful to better analyze MANEMO. Fifth, the wireless ranges, both in managed and ad-hoc mode, were limited in this evaluation, as the MRs and antenna were installed inside the vehicles. In future developments, vehicles should be equipped with outside antennae to achieve longer distance communications.

\section{Acknowledgment}

The authors would like to thank the ANEMONE project and partners, and more specifically ENST-Bretagne and INRIA Rennes who built the IPv6 mobility testbed from which the experiments exposed in this paper have largely benefited.

\section{REFERENCES}

[1] T. Ernst. The Information Technology Era of the Vehicular Industry. ACM SIGCOMM Computer Communication Review (CCR), Volume 36(Issue 2), April 2006.

[2] V. Devarapalli, R. Wakikawa, A. Petrescu, and P. Thubert. Network Mobility (NEMO) Basic Support Protocol, January 2005. IETF RFC3963.

[3] P. Jacquet T. Clausen. Optimized Link State Routing Protocol (OLSR), October 2003. IETF RFC3626.

[4] L. Bokor, N. Montavont, P. Di Francesco, and T. Ernst. Anemone : a pan-european testbed to validate ipv6 mobility technologies. 2007. The 2007 International Symposium on Applications and the Internet, Workshop on Network Mobility (SAINT WONEMO 2007), Hiroshima, Japan.

[5] T. Ernst, N. Montavont, R. Wakikawa, C. Ng, and K. Kuladinithi. Motivations and Scenarios for Using Multiple Interfaces and Global Addresses, July 2007. IETF, draft-ietf-monami6-multihoming-motivationscenario-02.
[6] C. Ng, T. Ernst, E. Paik, and M. Bagnulo. Analysis of Multihoming in Network Mobility Support, October 2007. IETF, RFC4980.

[7] R. Wakikawa, T. Ernst, K. Nagami, and V. Devarapalli. Multiple Care-of Addresses Registration, January 2008. IETF, draft-ietf-monami6-multiplecoa-05.

[8] D. Johnson, C. Perkins, and J. Arkko. Mobility Support in IPv6, June 2004. IETF RFC 3775.

[9] C. Ng, P. Thubert, M. Watari, and F. Zhao. Network Mobility Route Optimization Problem Statement, July 2007. IETF RFC4888.

[10] C. Ng, F. Zhao, M.Watari, and P. Thubert. Network Mobility Route Optimization Solution Space Analysis, July 2007. IETF RFC4889.

[11] R. Baldessari, A. Festag, and M. Lenardi. C2C-C Consortium Requirements for NEMO Route Optimization, July 2007. IETF, draft-baldessari-c2ccc-nemo-req-01.

[12] W. Eddy, W. Ivancic, and T. Davis. NEMO Route Optimization Requirements for Operational Use in Aeronautics and Space Exploration Mobile Networks, December 2007. IETF, draft-ietf-mext-aero-reqs-00.

[13] R. Wakikawa, K. Okada, R. Koodli, A. Nilsson, and J. Murai. Design of Vehicle Network: Mobile Gateway for MANET and NEMO Converged Communication, September 2005. VANET 2005.

[14] J. Lorchat and K. Uehara. Optimized Inter-Vehicle Communications Using NEMO and MANET (Invited Paper), July 2006. V2VCOM 2006.

[15] R. Wakikawa, T. Clausen, B. McCarthy, and A. Petrescu. MANEMO Topology and Addressing Architecture, July 2007. IETF, draft-wakikawa-manemoarch-00.

[16] R. Wakikawa, P. Thubert, T. Boot, J. Bound, and B. McCarthy. Problem Statement and Requirements for MANEMO, July 2007. IETF, draft-mccarthy-manemo-configuration-problems-01.

[17] C. J. Bernardos, I. Soto, M. Calderón, F. Boavida, and A. Azcorra. Varon: Vehicular ad hoc route optimisation for nemo. Comput. Commun., 30(8):1765-1784, 2007.

[18] C. Pradalier, J. Hermosillo, C. Koike, C. Braillon, P. Bessière, and C. Laugier. The cycab: a car-like robot navigating autonomously and safely among pedestrians. Robotics and Autonomous Systems, 50(1):51-68, 2005.

[19] M. Tsukada and T. Ernst. Vehicle Communication Experiment Environment With MANET And NEMO, January 2007. The 2007 International Symposium on Applications and the Internet, Workshop on Network Mobility (SAINT WONEMO 2007), p. 45, Hiroshima, Japan.

[20] H. Soliman, N. Montavont, N. Fikouras, and K. Kuladinithi. Flow Bindings in Mobile IPv6 and Nemo Basic Support, November 2007. IETF, draft-soliman-monami6-flow-binding-05.

[21] C. Larsson, M. Eriksson, K. Mitsuya, K. Tasaka, and R. Kuntz. Flow Distribution Rule Language for Multi-Access Nodes, November 2007. IETF, draft-larsson-mext-flow-distribution-rules-00. 